\title{
Modeling and Verification of Accumulators using CFD
}

\author{
Victor Irizar, Peter Windfeld Rasmussen, Olivier Doujoux Olsen*, and Casper Schousboe Andreasen* \\ Fritz Schur Energy A/S, Fabriksparken 7, 2600 Glostrup, Denmark \\ E-mail: vie@fsenergy.dk, pwr@fsenergy.dk \\ *Department of Mechanical Engineering, Solid Mechanics, Technical University of Denmark, Nils Koppels Alle, B.404, 2800 \\ Kgs. Lyngby, Denmark \\ *E-mail: s100196@student.dtu.dk, csan@mek.dtu.dk
}

\begin{abstract}
Hydraulic pitch systems provide robust and reliable control of power and speed of modern wind turbines. During emergency stops, where the pitch of the blades has to be taken to a full stop position to avoid over speed situations, hydraulic accumulators play a crucial role. Their efficiency and capability of providing enough energy to rotate the blades is affected by thermal processes due to the compression and decompression of the gas chamber. This paper presents an in depth study of the thermodynamic processes involved in a hydraulic accumulator during operation, and how they affect the energy efficiency of the component. An initial evaluation of the popular thermal time constant model is made and compared with experimental results for a 6-liter accumulator, showing that the current estimation techniques for the thermal time constant are not suited for the application studied, predicting higher heat loses in the gas and resulting in lower pressure buildup. Furthermore, it is shown that the assumption of a constant value for the thermal time constant can provide extremely accurate results, provided that the compression ratios of the process are known in advance. For varying compression ratios, dynamical effects play an important role and the accuracy of the model decreases. To study the thermal processes, a simplified axisymmetric CFD model of the accumulator is developed, using COMSOL Multiphysics for meshing and STAR-CCM+ as a solver. The model provided an interesting close up view to the gas movement and temperature distributions during operation, which describe a particularly nonlinear behavior of the heat losses and the thermal time constant. The model was successfully validated with experimental data, and provides a repeatable and accurate prediction of the gas states, regardless of the operational conditions, with maximum prediction errors of $10 \%$. Finally, a practical approach on how to improve the thermal efficiency of the accumulators by introducing foams on the gas side is shown, effectively decreasing the heat losses in the accumulator, and improving the efficiency of the compression-expansion cycles.
\end{abstract}

Keywords: CFD, Hydraulic Accumulators, Heat Transfer, Thermal Time Constant

\section{Introduction}

In modern pitch controlled wind turbines, hydraulic systems play an important role regulating their power production, e.g. by controlling the pitch angle of the blades [1]. In general, these systems consist of a hydraulic pump that supplies the oil flow, which is then routed by a set of control valves to a linear actuator attached to the blade (in one or two anchor points on the blade root) and its linear displacement is converted to an angular rotation of the blade. The purpose of the hydraulic control system is to ensure an efficient power production and to protect the turbine from dangerous situations like over-speeding or blade failures. When these emergency situations arise, the hydraulic system has to be able to pitch the blades up to a position where the turbine stops, mean- ing turning the blades to 90 degrees compared to the wind direction, as fast and reliable as possible. Hydraulic pitch systems include accumulators that store energy during normal operation, in terms of compressed gas (see fig. 1), and when the system performs an emergency stop, the accumulators provide high pressure flow to the actuators so the blade can be turned to the desired stop position.

In an ideal situation, all the energy stored in the accumulator can be utilized and delivered to the hydraulic system without thermal losses at the gas side during compression and expansion, but in reality, the process is not adiabatic. The gas experiences pressure and temperature variations with changes in volume, thus heat is transfered to the solid parts of the accumulator, and consequently to the environment, either through 
convection, conduction or radiation.

The complexity of these phenomena has been studied and modeled with empirical correlations that provide fairly accurate results for very specific situations [2-5], but little is reported about what is truly happening in the gas side of the accumulator. A better understanding of the thermo fluid dynamics of the gas in the accumulator can help improve current linear models, ultimately leading to optimization on the pitch control systems of the wind turbines, for example by reducing the size or the number of accumulators used per blade.

\section{Problem Description}

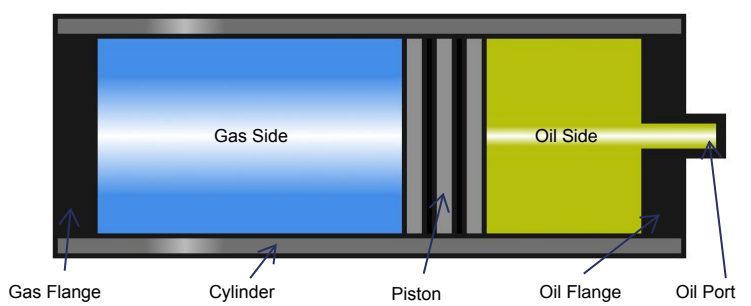

Figure 1: Basic layout of a piston type hydraulic accumulator

A general layout of a hydraulic accumulator is presented in fig. 1. The oil side has an input port through which the hydraulic oil can flow in or out. A floating piston separates the gas side from the oil. The gas is entrapped in the cylinder and is compressed by the piston when the oil starts flowing into the accumulator.

As the gas is compressed, its temperature and pressure rise. Equivalently, when the hydraulic system demands pressurized oil, the fluid leaves the accumulator and the gas expands, decreasing both its temperature and pressure. Heat transfer occurs between the gas and the solid parts of the accumulator, through convective and conductive mechanisms. The rate at which the heat transfer occurs is dependent on factors like the properties of the gas, pressure, temperature, initial conditions and the speed of the volume changes.

The challenge relies on how to model accurately and in a simplified way the state of the gas side and the heat losses to the surroundings, in order to predict the pressure and temperatures correctly, for different dimensions of accumulators and working conditions.

\section{The Thermal Time Constant Model}

For transient simulations, the mathematical modeling of a gas charged accumulator is often based on the thermal time constant model $[2,3,6]$.

This model is based on the assumption that the gas behaves as a lumped mass, which has constant properties along its domain, and it exchanges heat with the environment directly, by means of convection. Following this model, the rate of change of temperature in the gas can follows the following relation ${ }^{1}$

$$
\frac{d T}{d t}=\frac{T_{a}-T}{\tau}-\frac{T}{C_{V}}\left(\frac{\partial p}{\partial T}\right)_{V} \frac{d v}{d t}
$$

where $T$ is the bulk gas temperature, $T_{a}$ is the temperature of the surroundings (which are assumed constant), $\tau$ is the thermal time constant, $C_{v}$ is the specific heat capacity of the gas, $\frac{\partial p}{\partial T}$ is the partial derivative of the pressure w.r.t. the temperature (which is known from the equation of state) and $\frac{d v}{d t}$ is the rate of change of specific volume (volume per unit of mass). Furthermore, tau can be defined as

$$
\tau=\frac{m C_{V}}{h A}
$$

where $m$ is the mass of the gas, $h$ is the overall heat transfer coefficient from the gas to the surroundings and $A$ is the area of the heat transfer.

In order to make this model work accurately, first, a proper equation of state has to be chosen to couple the pressure and temperature states of the gas. Hansen and Rasmussen [1] proved that the Soave-Redlich-Kwong (SRK) equation yields excellent results for predicting the gas states in an hydraulic accumulator.

Second, a proper value or expression for $\tau$ has to be chosen, to predict accurately the heat losses. A set authors have developed expressions for estimating the parameter $[4,5,7]$, but these expressions are usually difficult to generalize when the accumulator dimensions and conditions change.

Looking deeper into the definition of $\tau$, several things can be noted

- It is directly proportional to the mass $m$ which means that is influenced directly by initial conditions like precharge pressure, temperature and initial volume

- It is inversely proportional to the heat transfer coefficient $h$, which includes the average contribution of the heat transfer processes from the gas to the wall of the accumulators, and from the accumulator outer walls to the environment. The internal heat transfer coefficient, however, is heavily influenced by the dynamics of the gas as well as it temperature and pressure, which implies that the thermal time constant is probably not "constant" during operation.

To have a in depth look of the fluid and heat transfer phenomena of the gas in the accumulator and better predictive performance, CFD modeling can provide a more accurate answer and understanding.

\section{CFD Modelling}

In order to study in depth the thermodynamics of the gas in the accumulator, a CFD model was developed. For the modeling

\footnotetext{
${ }^{1}$ The formal derivation of the model is omitted in this article for the sake of compactness, but the works of [2] and [1] provide the details
} 
of the accumulator, the use of two softwares was implemented. For the CAD modeling and meshing, COMSOL Multiphysics was used due to its powerful and flexible mesher and STAR CCM+ was then used for solving and post-processing of the results.

\subsection{Geometry}

The accumulator has the advantage of being of a symmetrical cylindrical shape, so to minimize the computational effort, a three dimensional wedge of the cylindrical domain was modeled, to be able to exploit the axis-symmetrical properties of the cylindrical shape of the accumulator. This wedge has an angular length of four degrees as seen in fig. 2 .

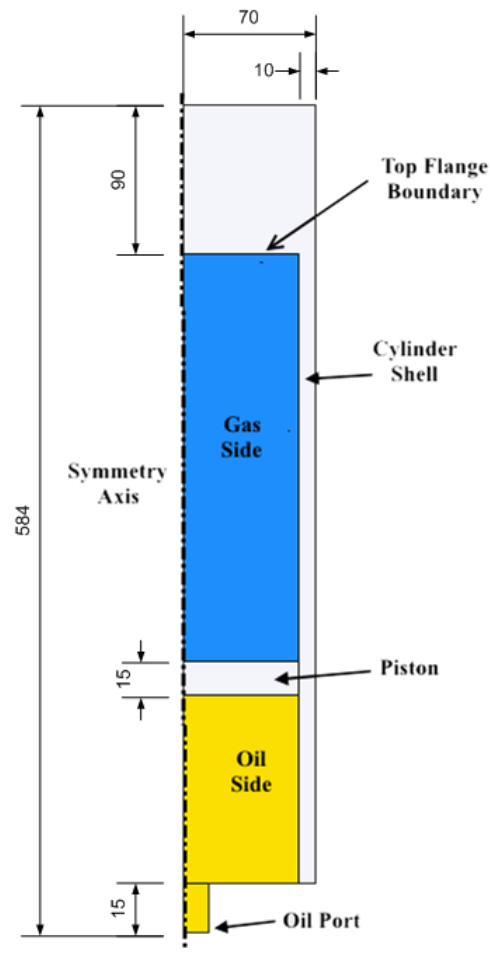

(a) Front View. Measurements in $\mathrm{mm}$.

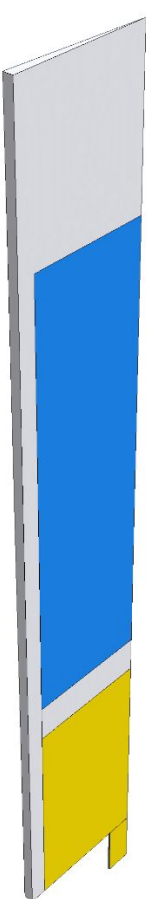

(b) Isometric View
Figure 2: CAD Model of the accumulator with differentiated colored domains (yellow for oil, blue for gas and grey for steel).

The main geometry is divided in 5 sub domains which are grouped by the type of physics modeling involved in them. Grey parts are solid sections made of steel which are the piston, cylindrical shell and top flange. The yellow domains are the oil side parts, which are the inlet port and the oil cavity. Finally the blue domain represents the gas chamber. The dimensions of the model are taken from CAD models provided by the accumulator manufacturer.

\subsection{Assumptions}

- Simplified geometry and reduced dimensions

- Simple lumped convective heat transfer through convection from the cylinder walls to the environment, of the form $Q_{\text {ambient }}=h_{\text {air }}\left(T_{\text {wall }}-T_{\text {ambient }}\right)$, where $T_{\text {ambient }}$ is a known measurable parameter from the experiments and $h_{\text {air }}$ is the convective heat transfer coefficient of stall air, which is set to $10 \mathrm{~W} / \mathrm{m} \cdot \mathrm{K}$ based on [8]

- No radiative heat transfer modeled, since the walls of the accumulator are usually below $40^{\circ} \mathrm{C}$, and the contribution is negligible [8].

- Oil domain modeled, considered relevant for heat transfer through the piston

\subsection{Solver Setup}

The transient simulation is solved by a fixed step implicit solver, with a time step of $0.001 \mathrm{~s}$, and first order discretization in time. The inner solver steps are performed in a segregated manner, where the momentum, pressure, energy and turbulence equations are solved one after the other. The inner solver loops until the residuals of the main quantities fall below a predefined tolerance (below $10^{-4}$ ). The differential equations are solved by a multi-grid method.

\subsection{Regions and Domains}

The model contains 3 main physical regions, corresponding to the 3 different material domains that can be found in the accumulator: Liquid for the oil domains, Gas for the accumulator's gas chamber and Solid regions for the remaining steel parts of the accumulator. Each region has an associated set of physical models selected, depending on the simulation needs.

In the gas region, the material used is diatomic Nitrogen $N_{2}$, which is built into the software's material database. Viscosity, thermal conductivity and specific heat capacity are considered functions of pressure and temperature. Force of gravity is enabled in the direction negative of the symmetry axis of the model. The turbulence model $k-\varepsilon$ is chosen based on computational efficiency and high accuracy for fully turbulent flows. A turbulence intensity of $4.5 \%$ and a turbulent viscosity ratio of 25 , based on [9]. The real gas equation of state used is Soave-Redlich-Kwong.

In the liquid region, material used is Hydraulic Oil based on the specifications of Castrol AWHM 32 [10] . Viscosity, thermal conductivity and specific heat capacity are considered constant. Gravity force is neglected and the flow is considered laminar since it is a high viscosity fluid. The oil is also considered isothermal.

In the solid region, material used is Carbon Steel based on the specifications of the manufacturer of the accumulator. Constant thermal conductivity of $k=49.8 \mathrm{~W} / \mathrm{mK}$ and specific heat capacity of $C_{p}=4900 \mathrm{~J} / \mathrm{kgK}$ were chosen.

\subsection{Mesh}

Based on the simplicity of the geometrical model, a mapped mesh with quadrilateral elements was chosen for the fluid domains, and a free triangular mesh was chosen for the rest of the solid parts of the geometry, which adapts to parameters selected for the fluid domains. These parameters control the number of elements present in the gas and oil domains to achieve the highest quality possible during the deformation 
and displacements of the domains. For accurate turbulence modeling, 10 boundary layers were added to the inner side of the boundaries of the gas domain. A close up of the mesh can be seen in fig. 3 .

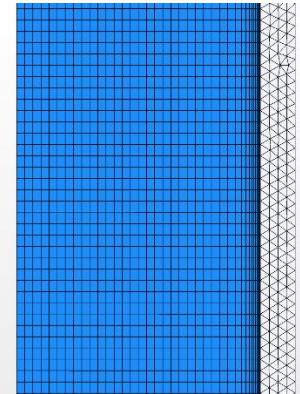

(a) Gas and Cylinder Shell

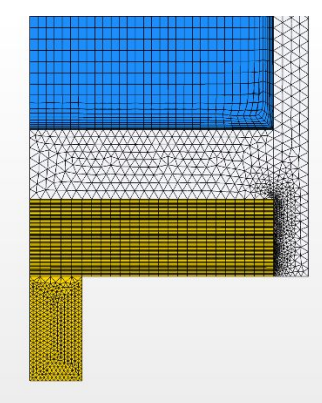

(b) Oil, Piston, Gas and Cylinder Shell
Figure 3: Detail of the model's mesh. Final iteration, number of elements: 4235.

The mesh size and setup was optimized to provide the highest accuracy and smallest level of distortion in the gas side mesh. The mesh deformation compresses and stretches the elements of the gas and oil domains, but there is no re-meshing step in between the solving steps.

\subsection{Calculation of Pressure and Temperature}

The CFD model includes a different set of probes and sensors to calculate the relevant properties and be able to truly compare with the real measurements in the experimental setup. The gas pressure is calculated as a mass average of the pressure in every cell. The average temperature is also calculated as a mass average of the individual temperature of all the cells. In addition to that, temperature probes were set in the model following the drawing in fig. 5.

\subsection{Model Validation}

A validation was carried out using the experimental runs described by [1]. The data obtained from this test runs includes the piston position over time and temperature and pressure of the gas domain. The validation was carried out with data from 19 selected runs from the previous study. In general, the average maximum error in pressure between the experimental and simulated results is of 5\%, and the average overall RMS error is of $2 \%$, which demonstrates that the model generates repeatable and reliable results for the range of conditions used in the previous experimental studies. The source of the errors that can be seen between the experimental and the simulated data can be explained by different factors. First, the reduced nature of the model, which neglects geometrical features and assumes symmetric flows (no swirl). Second, the uncertainties of experimental data, which include the noise in the signals, the reliability of the sensor positioning and the filtering techniques. And third, the numerical errors introduced by the deformation of the mesh in the CFD model and the degraded cell quality in some regions.

\section{Experimental Setup}

In order to asses the accuracy of the CFD model, an experimental validation was carried out. The experimental procedure and test equipment used in [1] served as a base for the validation carried out in this work. The gas temperature and pressure measured on a 6 liter accumulator subjected to compression-expansion processes were compared to the predictions of the developed CFD model. A diagram of the ex-

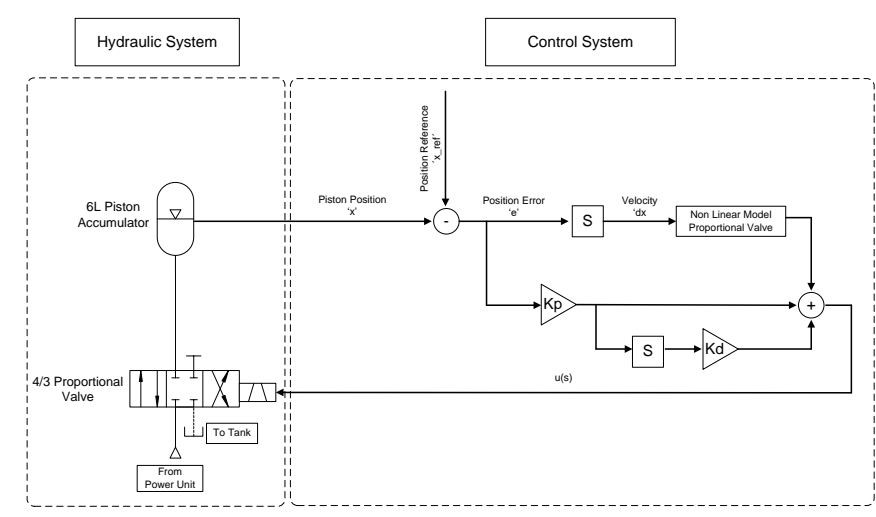

Figure 4: Hydraulic diagram of the experimental setup used for validation

perimental setup used can be seen in fig 4, with pictures of the main components presented in fig 6. The experimental setup consists of a test accumulator, mounted in a pivoting support to allow orientation changes (with the possibility to be rotated by 180 degrees from a vertical position). Oil is supplied to the test rig by an hydraulic power supply composed of two pumps and 4 accumulators. The supply is capable of delivering hydraulic oil at a maximum pressure of 250 bar.

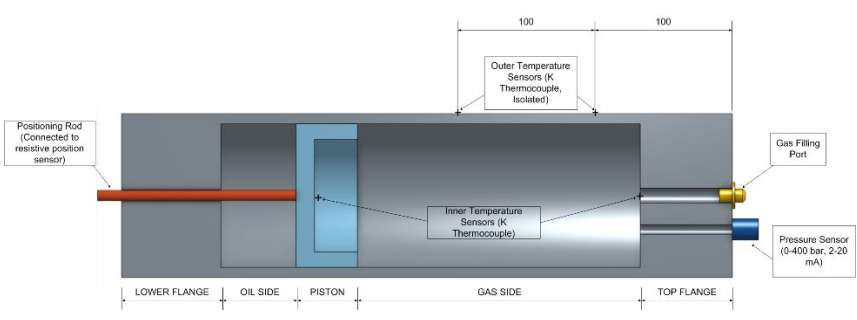

Figure 5: Basic schematic of sensor positioning in the test accumulator

The oil flow into the accumulator is controlled by a $4 / 3$ proportional valve. The position of the piston is controlled via a P-I controller, which receives the position signal of the piston and adjusts the flow through the hydraulic valve accordingly, using also a feed-forward loop on the velocity of the piston. The test accumulator is connected to a variety of sensors to monitor pressure and temperature at different points. Figure 5 shows the sensor positioning in the accumulator. The gas chamber is connected to a 400 bar pressure transmitter. In addition to that, two temperature sensors ( $\mathrm{K}$ type thermocouples) are positioned in the gas side, one in the center of the piston and the other on the top flange. Four more probes monitor the outer shell temperature (only two shown in the 


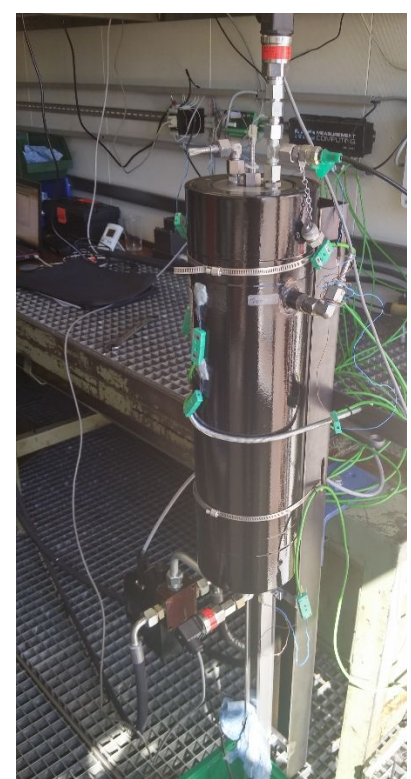

(a) Test Accumulator

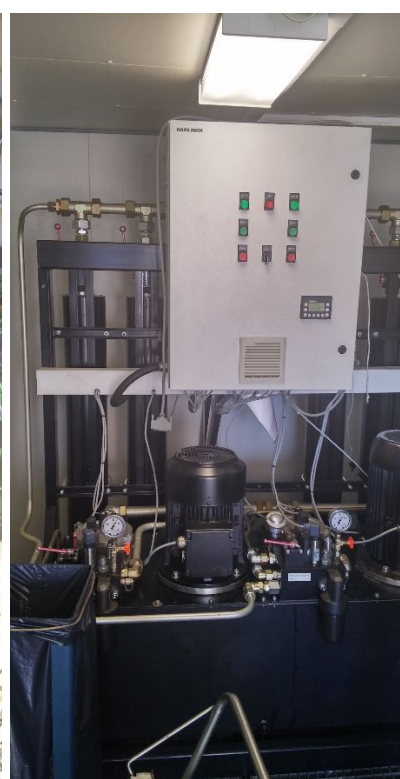

(b) Hydraulic Supply
Figure 6: Main hydraulic components of the experimental rig

figure, two extra sensors are positioned in the same axial coordinates but with a radial offset of 90 degrees). The piston is attached to a rod that drives the resistive position sensor, which lies outside of the accumulator.

All the sensors are connected to a data acquisition system which is itself linked to a main computer equipped with DASYLab software for data acquisition.

\begin{tabular}{ccc}
\hline$p_{0}[\mathrm{bar}]$ & $d V / d t[\mathrm{~L} / \mathrm{s}]$ & $V_{\text {fin }} / V_{\text {init }}$ \\
\hline 20 & {$[0.31,0.62,0.93]$} & {$[1.5,2.1,2.7]$} \\
50 & {$[0.31,0.62,0.93]$} & {$[1.5,2.1,2.7]$} \\
100 & {$[0.31,0.62,0.93]$} & {$[1.2,1.4,1.5]$} \\
150 & {$[0.31,0.62,0.93]$} & {$[1.1,1.15,1.2]$} \\
\hline
\end{tabular}

Table 1: Experimental setups for the tests made at Fritz Schur Energy. A three digit labeling system for the experimental runs is used to identify the different levels at which the above mentioned conditions can vary (ex. run $311=$ Initial Pressure Level 3, Volume Change Rate Level 1, Compression Ratio Level 1).

The accumulator is subjected to compression-expansion cycles where the pre-charge pressure, compression/expansion velocity and compression ratio were varied, based on the experiments of [1]. A three digit labeling system for the experimental runs is used to identify the different levels at which the above mentioned conditions can vary, following tab. 1 , for a total of 36 experimental cycles.

\section{Results And Comparison}

For this section, a compression-expansion case is studied which is based on the initial conditions of the run 233 de- scribed in tab. 1. The objective is to illustrate the dynamics of the system. The simulation is initialized with a temperature of $33.6^{\circ} \mathrm{C}$ and a pre-charge pressure of $50 \mathrm{bar}$, with an initial volume of 5.21 liters and a compression ratio of 2.7. Figure 7(a) shows the volume of the gas chamber during the process. The accumulator is oriented vertically.

For the CFD model, the reference signal of the volume changes in time were used. Unusual behavior of the heat transfer in the system was experienced when the signal inputed into the CFD model had some level of noise (when, for example, the measured piston position from the experiments was used as an input to the piston position in the CFD model). Large amounts of heat could be seen transfered from the gas to the walls of the accumulator. This sensitivity can be a consequence of the choice of the turbulence model selected. The $k-\varepsilon$ turbulence model assumes that turbulent stability is achieved, and the noise in the piston movement can excite the system enough to create a highly turbulent behavior near the walls, causing excessive heat loss and unreal results.

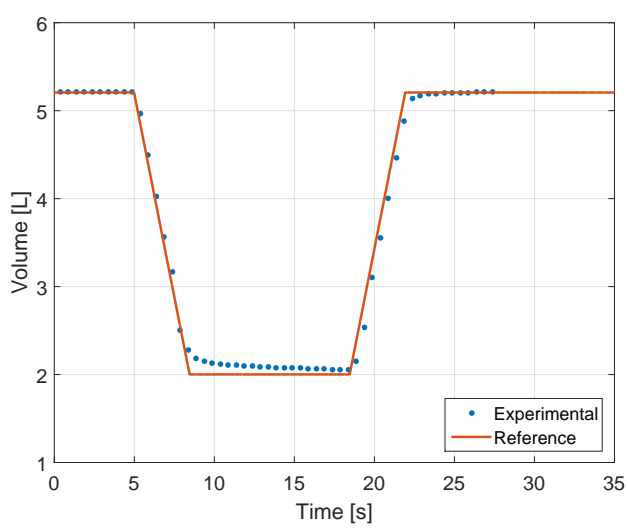

(a) Gas Volume

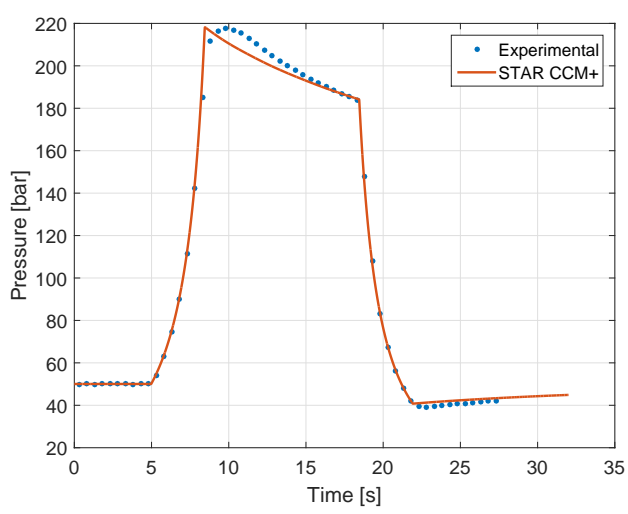

(b) Gas Pressure

Figure 7: Gas volume and pressure for the compressionexpansion cycle

The pressure evolution obtained by the CFD simulation has a good level of agreement with the experimental reference, fig. 7(b), which provides a solid validation point for the model. The discrepancies may originate from the way the position of the piston is controlled in the experimental setup, which is equivalent as filtering the input signal. This smoothing is omitted in the CFD model due to the fact that it would 
require the measured piston position from the experiments, which contains noise and can produce unreliable results due to the issues explained above.

\subsection{Temperature Distribution}

Figure 8 shows the evolution of the temperature distribution in the accumulator for the compression-expansion cycle. The compression process starts at $t=2.0$ seconds, the gas increases temperature due to the reduction of the volume, however the regions close to the cylinder shell remain at lower temperatures due to the heat exchange with colder solid domain. At $t=4.0$ seconds, a vortex of colder gas can be seen emerging from the piston, revolving clockwise towards the walls of the accumulator. The movement of the piston drags the colder gas in the boundary layer at the inner wall of the cylinder, and is pushed inwards into the centerline of the domain, passing above the piston. From $\mathrm{t}=6.0$ seconds to $\mathrm{t}=$ 14.0 seconds the compression is held. The gas reaches a maximum temperature of $184{ }^{\circ} \mathrm{C}$, which decreases over time due to the diffusion and natural convection. It can be seen that the temperature is clearly stratified, from top to bottom of the gas domain. Temperature differences between the top of the gas domain and the bottom (where the piston lies) of up to $60^{\circ} \mathrm{C}$ are visible. The solid parts of the accumulator surrounding the gas domain also increase their temperature, up to $10{ }^{\circ} \mathrm{C}$, due to heat transfer from the hotter gas. At $t=16.0$ seconds, the expansion process has begun. The gas temperature near the piston decreases faster than the temperature at the top, as the gas expands faster due to the influence of the piston motion. Clear temperature gradients can be seen along the domain in the vertical direction as in the compression part. Now the heat transfer process is reversed, the solid parts of the accumulator are at a higher temperature than the gas. The shell of the accumulator remains at a higher temperature in the areas where the volume was held after the compression. The results show that temperature gradients along the gas domain exist. Experimental temperature measurements of the gas are influenced by the position of the sensor. A set of virtual probes were setup in the CFD model to compare with the experimental measurements.

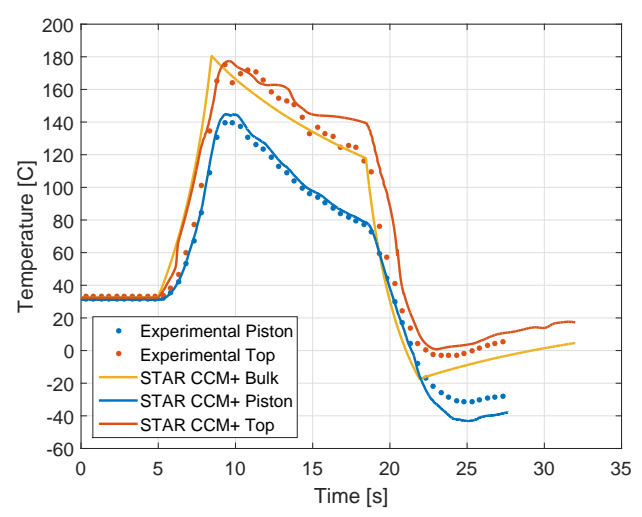

(a) Gas Domain Temperatures

Figure 9: Temperature measurements in the accumulator. Experimental vs. CFD results
Figure 9 shows the temperature measurements at different positions in the gas domain. As shown in the aforementioned results, the temperatures measured at the top of the domain are higher than the ones on the piston, due to the gradient imposed by the gravitational forces. Simulated results of the temperatures in the probes have fluctuations, a consequence of the fluid dynamics in the gas, when hotter and colder gas move near the probe. The simulated bulk (or average) temperature of the domain, calculated as the mass averaged temperature of the gas, lies in between the top and the piston temperatures, as it behaves as an average expression of the temperature in the whole domain. Experimental results show very good agreement with the simulated ones, and reflect the importance of choosing an appropriate measuring position of the temperature sensors in the experimental setup to have a good reference to compare simulation models. Measured temperatures from the top of the domain provide a good approximation to the average temperature of the gas.

\subsection{Heat Transfer Across Boundaries}

With the CFD model, the boundary fluxes in the walls of the gas domain can be integrated to obtain the heat transfered between bodies. The resulting rate of heat losses are presented in fig. 10(a).

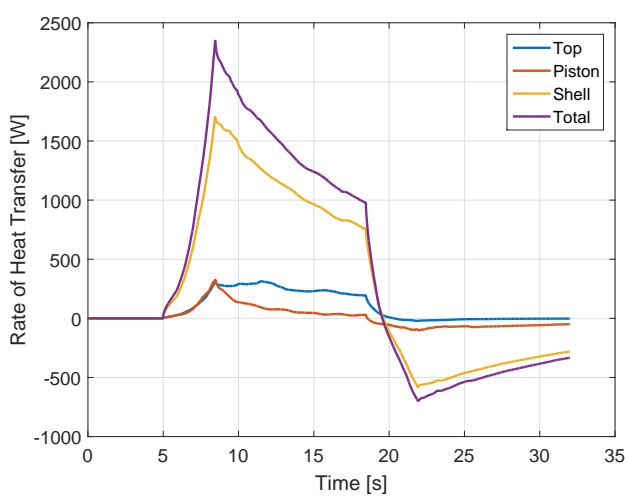

(a) Gas Domain Heat Loses

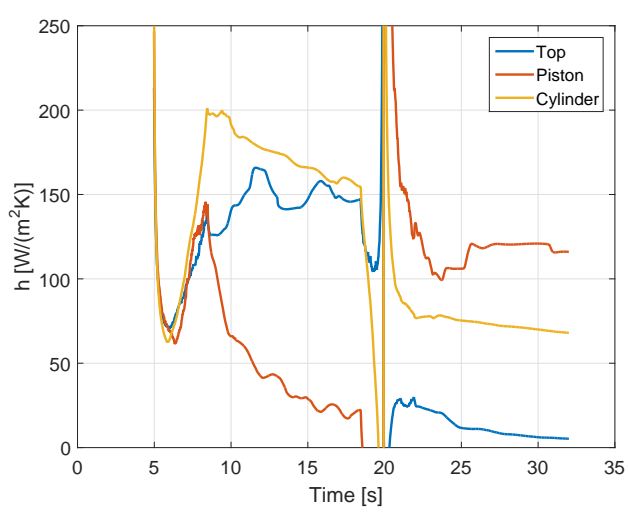

(b) Heat Transfer Coefficients

Figure 10: Boundary heat losses in the gas domain from the CFD simulation of the case studied.

It can be seen that the heat losses follow the dynamics of the compression-expansion cycle. Heat loss increases as the gas 

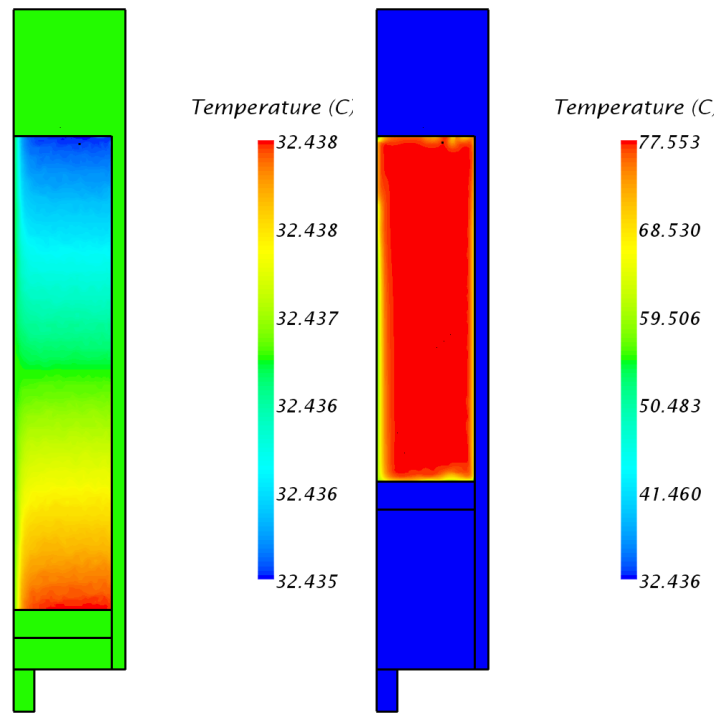

(b) $\mathrm{t}=1.0 \mathrm{~s}$
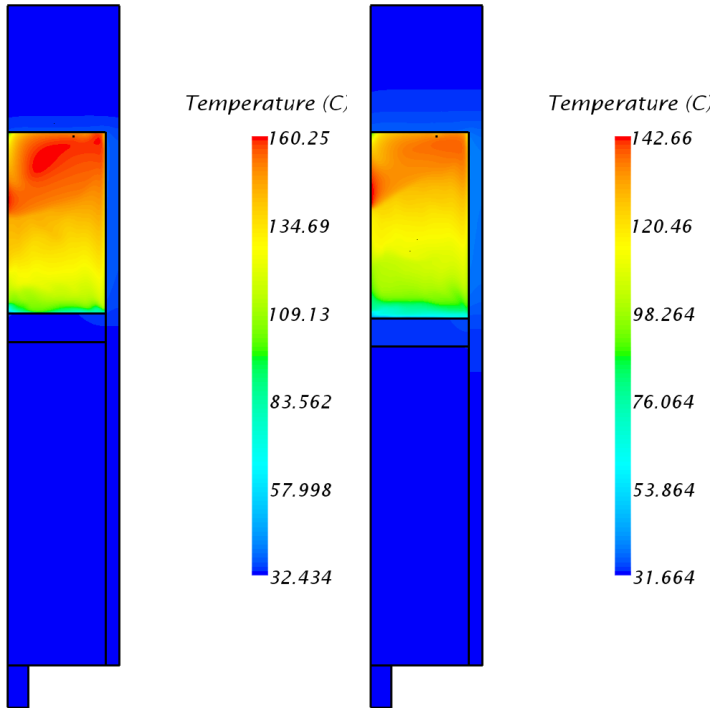

(e) $\mathrm{t}=10.0 \mathrm{~s}$

(f) $\mathrm{t}=14.0 \mathrm{~s}$
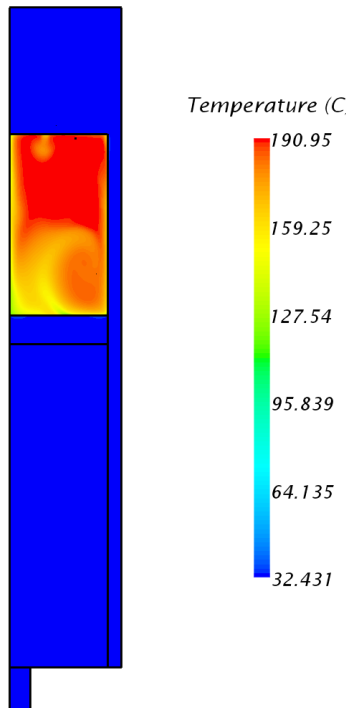

(c) $\mathrm{t}=4.0 \mathrm{~s}$

(d) $\mathrm{t}=6.0 \mathrm{~s}$
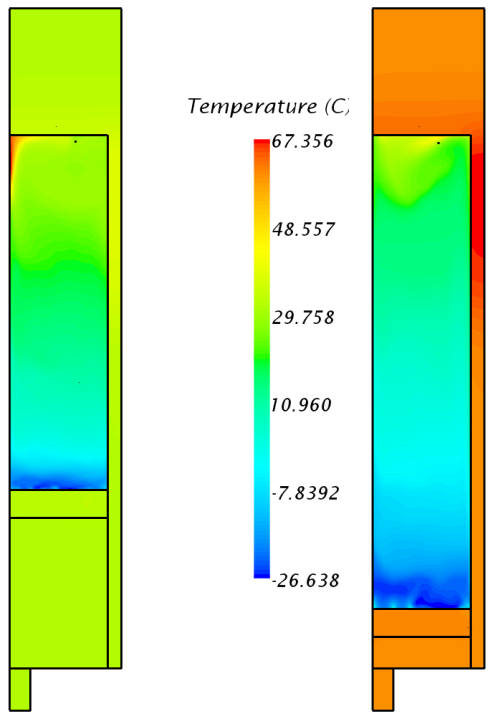

Temperature $(C$

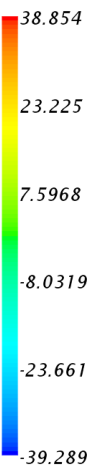

(g) $\mathrm{t}=16.0 \mathrm{~s}$

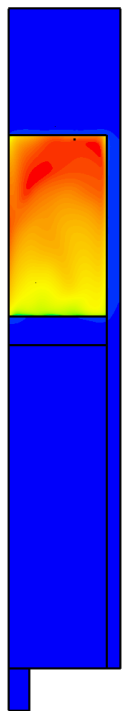

Temperature $(C$

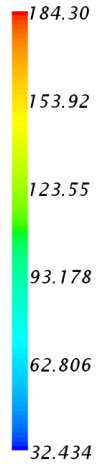

(h) $\mathrm{t}=18.0 \mathrm{~s}$

Figure 8: Temperature distribution in the accumulator during the compression-expansion cycle

is compressed due to the continual increase of temperature and the momentum induced in the gas due to the piston movement. After the compression, the heat transfer gradually decays due to a sustained decline of the gas temperature and the increase of temperature in the solid bodies of the accumulator. During the expansion, the heat transfer decreases gradually as the gas gets colder. At around 20 seconds into the simulation the heat transfer process reverses, now the gas receives heat from the hotter boundaries. When the expansion finalizes, the gas keeps receiving heat from the solid boundaries, and the magnitude of the heat transfer diminishes as time passes, due to the gas and the solid boundaries reaching slowly thermal equilibrium. Most of the heat is transfered through the cylinder shell boundaries. This responds to the fact that it has the biggest heat transfer area (which is between 10 and 2 times higher than the one of the piston or the top boundary) and high fluid velocities because of natural convection effects (as seen in fig. 11(a)). The heat losses through the piston and top boundary are smaller, accounting $15 \%$ of the total. It can be seen that for the top boundary the heat transfer process does not experience an inversion when the expansion begins. This is due to the small temperature difference between the gas and the boundary. In the piston, a small recovery can be seen after the compression. The lowest temperatures occur near this boundary so the temperature difference is high enough to be able to recover some of the heat.

The heat transfer and the corresponding heat transfer coefficients, associated to each boundary, (fig. 10(a) and 10(b)) show that after the compression, the highest heat transfer is experienced in the cylinder wall, while the lowest is in the 
piston. The temperature difference between the piston and the cold gas at the bottom of the domain is the smallest between the boundaries, which results in lower heat transfer. On the top boundary, the higher gas temperatures are found, which increases the heat transfer coefficient. After the expansion, the piston-gas interface has the highest values of $h$, a consequence of the higher temperature difference between the now colder gas at the bottom of the gas domain and the piston. Still, although having a reduced heat transfer coefficient, the highest amount of heat is transfered through the cylinder walls due to the large heat transfer area.

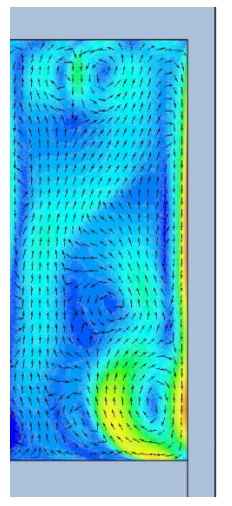

(a) Velocity Magnitude

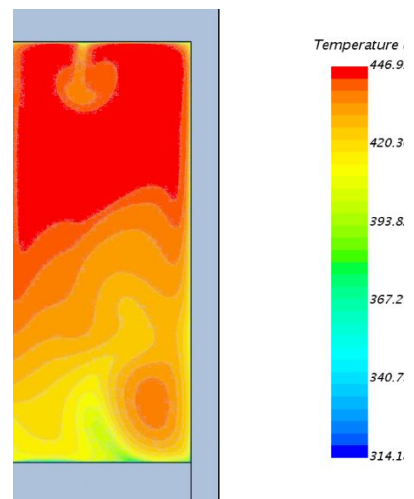

(b) Temperature
Figure 11: Velocity field and Temperature of the gas domain after compression ( $t=10$ seconds)

The curves represented in fig. 10(b) suggest that there is a clear non linear behavior of the heat transfer coefficient to the temperature difference between the gas and the surroundings. This implication has a direct influence in the way the heat losses are linearly modeled in eq. 1 , and specifically on how $\tau$ is conceived mathematically. Non linearities arise due to the fact that the fluid flow models are naturally non linear (incompressible Navier-Stokes, turbulence modeling), and the thermodynamical behavior of the system is influenced by the momentum of the flow and the local changes of viscosity and density, which themselves are non linear functions of the temperature.

\subsection{The Thermal Time Constant}

The dynamic behavior of the heat transfer phenomena in the gas domain can be expected to be reflected in the thermal time constant of the gas. Figure 12 shows the variations of the thermal time constant of the gas over time. Initially, as the process is initialized, the thermal time constant has a value of zero. This is due to the way the thermal time constant is mathematically defined to be calculated in the simulation. $\tau$ can be defined as

$$
\tau=\frac{m C_{V}\left(T_{s}-T\right)}{Q}
$$

where $Q$ is the heat loss across the boundaries of the gas domain and $T_{S}$ is the temperature of the walls (which now replaces $T_{a}$ since the gas in reality exchanges heat with the cylinder walls, and the temperature of these walls changes over time). When the compression starts, $\tau$ increases, and reaches a maximum value, and afterwards starts decreasing to its lowest value at the end of the compression. This behavior can be expected due to the fact that, when compression begins, the temperature increases first due to interactions of the gas molecules, and the heat transfer is low since there is no convection. After some seconds, the convective effects catch up with the increase in temperature, starting up the heat transfer to the boundaries, and lowering the thermal time constant.

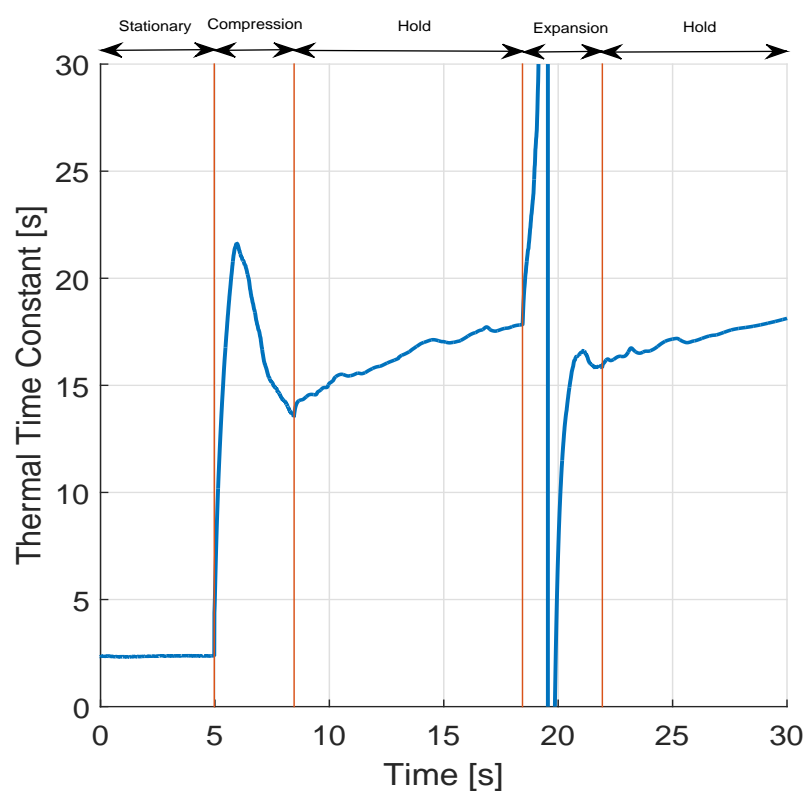

Figure 12: Thermal time constant evolution for a compression-expansion cycle

When the compressed gas is held, $\tau$ increases slowly, almost linearly. This corresponds to the steady heat transfer between the gas and the surrounding boundaries, which are slowly reaching thermal equilibrium. This behavior can also be seen after the expansion when the volume remains constant. During the expansion, the value of $\tau$ jumps from positive to negative suddenly. This is a result of how the time constant is defined mathematically. The denominator of eq. 3 is close to zero when there is no heat transfer, resulting in a visible discontinuity of the thermal time constant. This is a direct consequence of the temperature of the gas reaching lower temperatures than the boundaries due to the dilation of the chamber, and instantaneously the temperature difference is zero, thus also the heat exchange.

\section{Practical Approach for Improving the Effi- ciency of the Accumulator}

As seen from the CFD analysis, the behavior of the heat transfer in the gas is clearly non linear, and sensitive to several operational and dimensional factors. Although the CFD model provides a very good approach to a repeatable and accurate prediction of the losses in the accumulator, one may wonder if there is anything practical to be done to reduced the thermal losses in the accumulator.

Recalling the definition of $\tau$ in eq. 2, we can see that on 
practice, the two parameters that can be modified in a controlled matter are the gas mass $m$ and the heat transfer area $A$. The heat transfer area, though, can be tricky to modify since the size of the accumulator is usually defined based on the hydraulic system needs. Which leaves the mass as the only parameter that could be tweaked to increase performance. But how to do that?

Pourmovahed et al. [11, 12] investigated the influence of filling the gas side of the accumulator with elastomeric foams. Their research shows that, due to the porous nature of the foam, if one can effectively fill the whole gas compartment with it, the effect is the equivalent to increasing the heat capacitance of the gas, meaning that the term $m C_{v}$ in the $\tau$ definition is greatly increased due to the increased mass and heat capacitance, contributed by the foam. Inspired by this results, an experimental investigation was carried out.

\subsection{Experimental Setup}

The same experimental setup was used as with the CFD investigation. This time however the gas side was filled with two different types of foams: PPI 80 polyester (labeled as 'Foam 1' in the results) and HR 50 polyether (labeled as 'Foam 2' in the results). For each foam, a piece was custom made to fill the dimensions of the whole gas side, and was fixed to the piston of the accumulator to prevent it to get stuck during the compression and expansion phases. Figure 13 shows the foams used in the experiment, properly shaped and attached to the piston.

\begin{tabular}{ccc}
\hline$p_{0}[\mathrm{bar}]$ & $d x / d t[\mathrm{~mm} / \mathrm{s}]$ & $V_{\text {fin }} / V_{\text {init }}$ \\
\hline 50 & {$[50,100]$} & {$[1.50,2.00,2.50]$} \\
100 & {$[50,100]$} & {$[1.25,1.50,1.65]$} \\
\hline
\end{tabular}

Table 2: Experimental setups for foam tests made with a 6 liter accumulator.

For each foam filling, a set of experiments were carried out following the planning on tab. 2, Varying compression rations, velocities and pre-charge pressures.

\subsection{Results}

The use of PPI 80 polyester (foam 1) and HR 50 polyether foam (foam 2) generally showed positive results. The pressure curves of the work cycles, that are the most and the least effected by the foam filling, efficiency wise, are plotted in fig. 14. These curves give a quick overview of how the normal shaped foams affect the thermal time constant of the accumulator.

In the most effective case (fig. 14(a)) where the pre-load pressure is low, while the compression ratio is high, both foams have a remarkable effect on the pressure build up. The maximum pressure is reduced from 181 bar to 153 bar and 152 bar with foam 1 and 2 respectively. This means that less heat is lost, thus reducing the pressure drop during the holding period. The flat curves during the holding indicate a high $\tau$

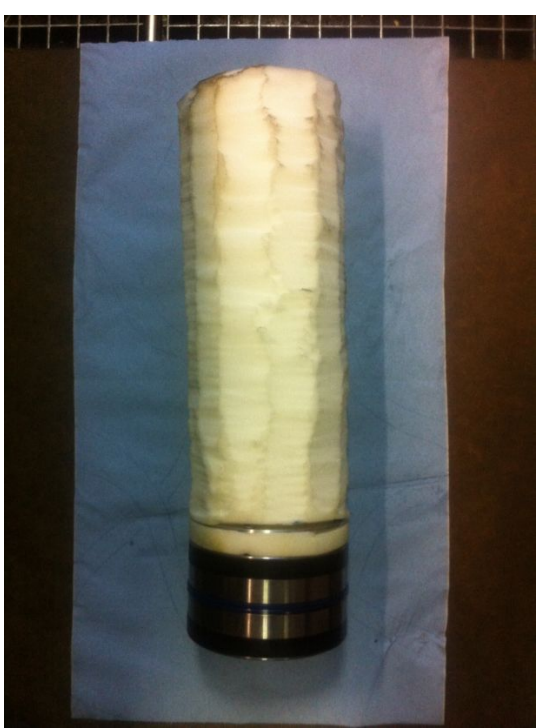

(a) Foam 1 shaped and attached to piston

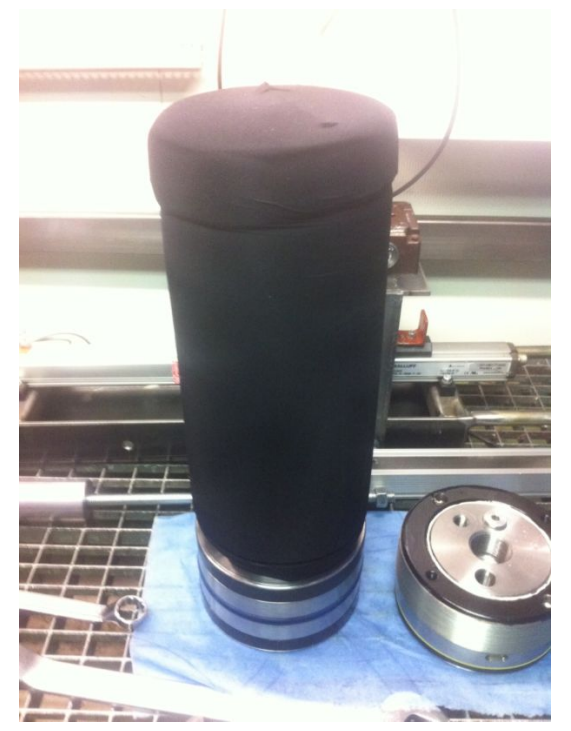

(b) Foam 2 shaped and attached to piston

Figure 13: Pictures of the filling foams used in the experiment

of the accumulator, which in this case is increased from $7 \mathrm{~s}$ without foam, to $46 \mathrm{~s}$ with foam 1 and $134 \mathrm{~s}$ with foam 2 .

In the least affected case shown (fig. 14(b)), the pre-load pressure is high while the compression ratio is small, and the piston speed is slow. This means that the overall pressure and temperature differences are lower, resulting in a more subtle improvement of $\tau$, which is now increased from $9 \mathrm{~s}$ without foam, to $34 \mathrm{~s}$ with foam 1 and to $74 \mathrm{~s}$ with foam 2 .

In both cases the thermal time constant is increased, and in both cases the highest increase is obtained with foam 2 . This is explained by the greater heat capacity, which is one of the main factors causing the improvements when using the foam. Since the heat capacity and density of the solid polyurethane is more or less the same for both foams, the heat capacity of foam 2 is larger, because the higher bulk density. When filling the gas chamber with foam, the effective volumetric heat ca- 


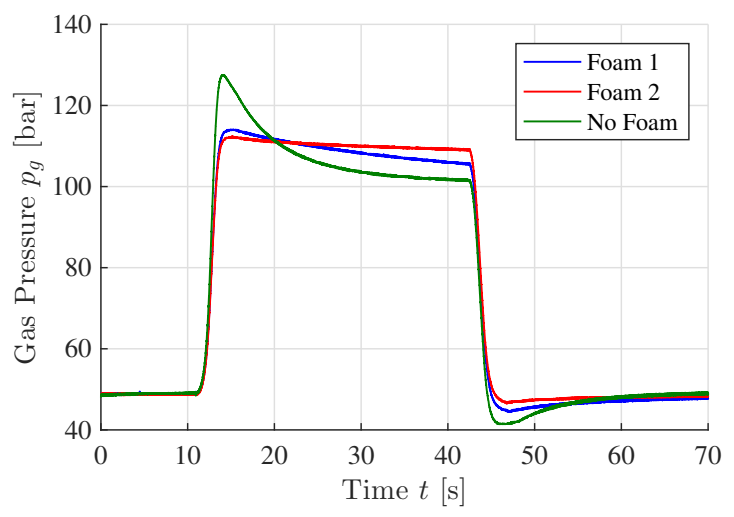

(a) $p_{0}=50 \mathrm{bar}, \mathrm{cr}=2.5, v_{p}=100 \mathrm{~mm} / \mathrm{s}$

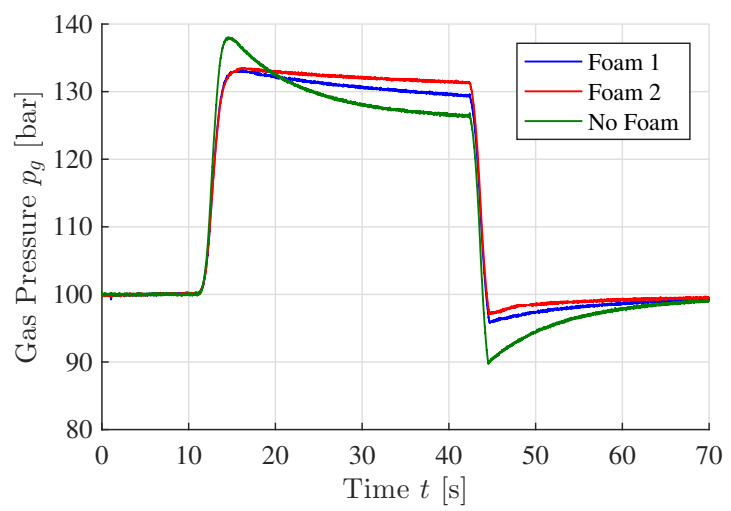

(b) $p_{0}=100$ bar, $\mathrm{cr}=1.25, v_{p}=50 \mathrm{~mm} / \mathrm{s}$

Figure 14: Accumulator pressures comparison for selected compression expansion cycles, with and without foam.

pacity of the gas and foam combination is higher compared to that of the gas alone. Because of the higher heat capacity, the foam works as a heat sink, which in turn reduces the temperature increase and thereby the pressure build up. Another positive effect of the foam is that gas flow is restricted due to the resistance of the porous structure. This will dampen the dynamics that are induced on the gas by the piston movement and further reduce the high temperatures that are seen in the accumulator without foam. As mentioned a lower maximum temperature means that less heat can be lost, and ultimately that the thermal efficiency is increased. In addition the restricted flow will reduce the convective heat loss to the cylinder walls.

\subsection{Reduction of Thermal Losses}

Since the main energy loss is the heat loss, the effect of the foam is best shown and explained with the pressure-volume $(\mathrm{pV})$ diagrams, that are shown in fig. 15 for the most and least effected runs respectively. The $\mathrm{pV}$ diagrams demonstrates graphically how the thermal losses, with and without foam, compares to each other and to the ideal case of isothermal compression.

The high compression ratio generates higher temperatures and pressure during compression. This is seen in the $\mathrm{pV}$ diagram in fig. 15(a), by the exponential increase of the pressure during compression. In contradiction, when the compression ratio is small, the slope of the $\mathrm{pV}$ diagram during these processes, is close to being linear and closer to the isotherm, as seen in fig. 15(b) After a large compression, the high temperature lead to greater heat losses during the holding period, which increases the pressure drop and in turn decreases the potential energy of the accumulator. This is represented by the vertical lines at the end of the compression process in the $\mathrm{pV}$ diagram. In addition to the big pressure drop at this point, a longer expansion makes the gas reach even lower temperatures and pressure at the end of the stroke. Ultimately this means, that the expansion work is done at much lower pressure than the compression work, resulting in the lowest possible energy efficiency and therefore the biggest improvement potential.

The foam reduces the heat loss by 3 mechanisms in 3 steps:

- Compression: The foam acts as a heat sink and dampens convection - reducing

- Holding period: The foam acts as a heat regenerator reducing temperature loss.

- Expansion: The foam acts as a heat regenerator and dampens convection reducing temperature decrease.

Since the foam works as a heat sink it is most effective when the temperature increase of the gas is high, namely for high compression ratios. A bigger step change of gas temperature, will cause a bigger temperature difference between the gas and the foam, which increases the heat transfer from gas to foam and thereby improves the effect of the foam. The regenerator effect is seen during the holding period and expansion process, where the gas temperature drops below that of the foam, and the heat transfer changes direction.

The effect of the foam is clearly seen by the reduction of the area inside the $\mathrm{pV}$ diagrams. And as seen with the thermal time constant the best overall effect is achieved with foam 2, namely the HR 50 polyether foam. In the most effective case shown in fig. 15(a) the heat loss of the accumulator is reduced with 23.8 percentage points from $30.8 \%$ without foam, to only $7.0 \%$ with foam 2 . And in the least effective case, shown in fig. 15(b), it is reduced by 8.4 percentage points, from $10.5 \%$ without foam to $2.1 \%$ with foam 2 .

\section{Conclusions}

The thermodynamical processes involved in an hydraulic accumulator during operation were successfully studied by the use of a CFD model. The results obtained from this model had a high level of agreement with the experimental results for a wide range of cases. The results demonstrate that the heat losses in the gas side of an accumulator are mainly associated with heat transfered to the solid parts that is later diffused into their bodies. The heat loses to the environment are relatively small for the conditions simulated, and have no meaningful effect in the system. There is also clear evidence that the there are important temperature gradients in the gas that make the heat losses not uniform throughout the boundaries. 


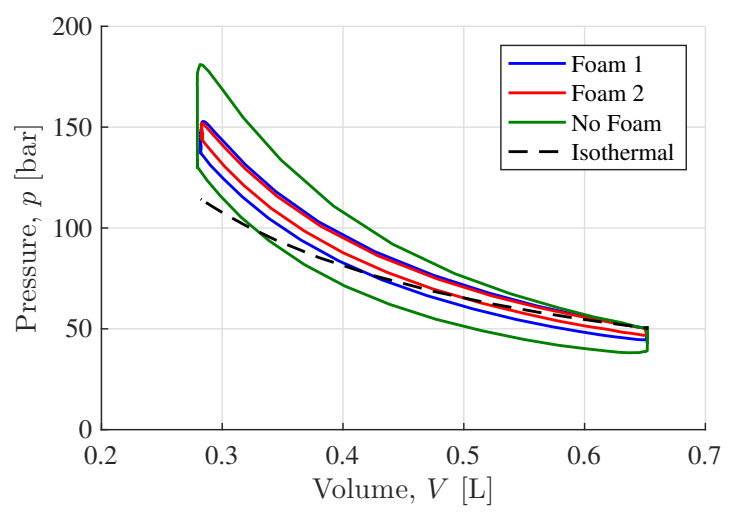

(a) $p_{0}=50 \mathrm{bar}, \mathrm{cr}=2.5, v_{p}=100 \mathrm{~mm} / \mathrm{s}$

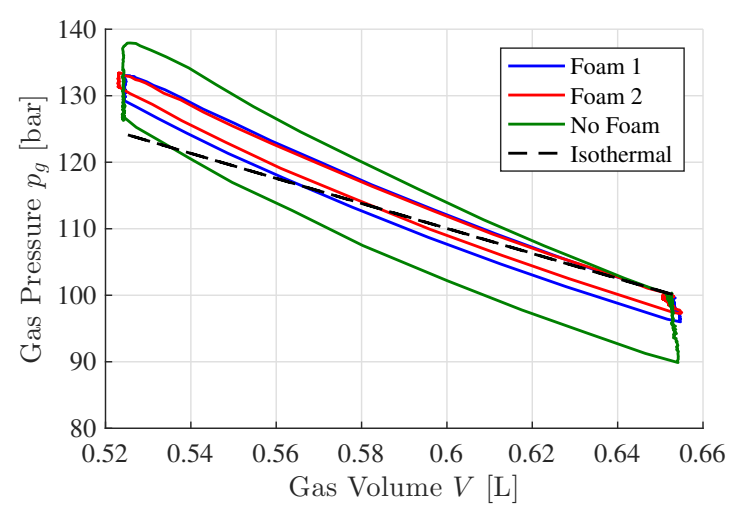

(b) $p_{0}=100$ bar, $\mathrm{cr}=1.25, v_{p}=50 \mathrm{~mm} / \mathrm{s}$

Figure 15: Accumulator $p-V$ diagrams comparison for selected compression expansion cycles, with and without foam, included also isothermal curves

It has been proved that the thermal time constant of the gas varies in time. It is proportionally dependent on the gas mass in the chamber, and is affected by changes in the initial conditions, orientation, compression velocity and compression ratio. The dynamics of $\tau$ are defined by the fluid flow of the gas. Convection is the main heat transfer process between the gas and the solid parts and it is affected by the piston movement, which induces momentum into the flow. The non linear behaviors of the heat transfer coefficients and thermal time constant are generated by the fluid motion and the convective processes.

A practical proof on how to improve the efficiency of the hydraulic accumulators has been presented and studied in depth, where filling the gas side with foams provides an increased heat capacitance of the gas and reduces the heat transfer to the accumulator walls, providing a better storage of the hydraulic power.

\section{References}

[1] H B Hansen and P W Rasmussen. Modelling hydraulic accumulators for use in wind turbines. Proceedings of the 13th Scandinavian International Conference on Fluid Power, Linköping, Sweden, 2013.
[2] D R Otis. Predicting performance of gas charged accumulators. Proceedings of the 1st Fluid Power and Control Systems Conference, University of WisconsinMadison:160-165, 1973.

[3] A Pourmovahed, N H Beachley, and F J Fronczak. Modeling of an hydraulic energy regeneration system - part i: Analytical treatment. Transactions of the ASME, 114:160-165, 1992.

[4] S Rotthäuser. Verfahren zur Berechnung und Untersuchung hydropneumatischer Speicher. Fakultät für Maschinenwesen der Rheinisch-Westfälische Technische Hochschule Aachen, 1993.

[5] K R Rupprecht. Hydrospeicher, Experimentelle und analytische Untersuchungen zur Energiespeicherung. Fakultät für Maschinenwesen der RheinischWestfälische Technische Hochschule Aachen, 1988.

[6] D R Otis and A Pourmovahed. An algorithm for computing nonflow gas processes in gas springs and hydropneumatic accumulators. ASME Journal of Dynamic Systems, 107:93-96, 1985.

[7] J. Svoboda, G. Bouchard, and S. Katz. A thermal model for gas-charged accumulators based on the heat conduction distribution. Fluid Transients and Acoustics in the Power Industry, pages 161-167, 1978.

[8] F Incropera, D DeWitt, A Lavine, and T Bergman. Fundamentals of Heat and Mass Transfer. John Wiley and Sons, 2011.

[9] J E Bardina, P G Huang, and T J Coakley. Turbulence modeling validation, testing, and development. NASA Technical Memorandum 110446., 1997.

[10] BP Marine Limited. Hyspin awh-m range oils. anti-wear hydraulic oils., October 19 2009. US Patent 2,331,921.

[11] A Pourmovahed, S Baum, N H Beachley, and F J Fronczak. Experimental evaluation of hydraulic accumulator efficiency with an without elastomeric foam. Journal of Propulsion and Power, 4:185-192, 1988.

[12] A Pourmovahed. Durability testing of an elastomeric foam for use in hydraulic accumulators. Journal of Solar Energy Engineering, 112:223-228, 1990. 